\title{
Potential Effects of Dietary Isoflavones on Drug-Induced Liver Injury
}

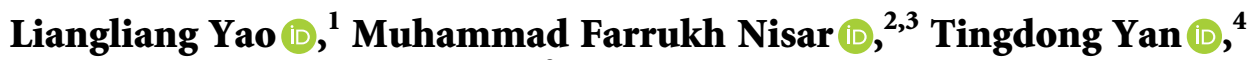 \\ and Chunpeng (Craig) Wan $\mathbb{D}^{2}$ \\ ${ }^{1}$ Affiliated Hospital of Jiangxi University of Traditional Chinese Medicine, Nanchang, Jiangxi 330006, China \\ ${ }^{2}$ Jiangxi Key Laboratory for Postharvest Technology and Nondestructive Testing of Fruits \& Vegetables, College of Agronomy, \\ Jiangxi Agricultural University, Nanchang 330045, China \\ ${ }^{3}$ Department of Physiology and Biochemistry, Cholistan University of Veterinary and Animal Sciences (CUVAS), \\ Bahawalpur 63100, Pakistan \\ ${ }^{4}$ School of Pharmacy, Nantong University, Nantong 226019, China
}

Correspondence should be addressed to Tingdong Yan; yantdntu2018@163.com and Chunpeng (Craig) Wan; chunpengwan@ jxau.edu.cn

Received 16 July 2021; Accepted 23 August 2021; Published 30 August 2021

Academic Editor: Shengbao Cai

Copyright $(92021$ Liangliang Yao et al. This is an open access article distributed under the Creative Commons Attribution License, which permits unrestricted use, distribution, and reproduction in any medium, provided the original work is properly cited.

Numerous prescribed drugs and herbal and dietary supplements have been reported to cause drug-induced acute liver injury, which is a frequent cause of acute liver failure (ALF). It is a tremendous challenge with ever-increasing drug application in the medication system for huge populations. Drug-induced acute liver injury can lead to diverse pathologies similar to acute and chronic hepatitis, acute liver failure, biliary obstruction, fatty liver disease, and so on. Recently, extensive work demonstrated that isoflavones play an essential and protecting role in drug-induced liver injury (DILI). The isoflavones mediated hepatoprotection by modulating specific genes linked with control of cellular redox homeostasis and inflammatory responses. Isoflavones upregulate oxidative stress-responsive nuclear factor erythroid 2-like 2 (Nrf2), downregulate inflammatory nuclear factor- $\kappa \mathrm{B}$ $(\mathrm{NF}-\kappa \mathrm{B})$ signaling pathways, and modulate a balance between cell survival and death. Moreover, isoflavones actively inhibit the expression of cytochromes P450 (CYPs) enzyme during drug metabolism. Moreover, isoflavones are also linked with farnesoid X receptor (FXR) activation and signal transducer and activator of transcription factor 3 (STAT3) phosphorylation in hepatoprotection DILI. In vivo and in vitro studies clearly stated that isoflavones bear strong antioxidant potential and promising agents for hepatotoxicity prevention and stressed their potential role as therapeutic supplements in DILI. The current review will elaborate on isoflavones' preventive and therapeutic potential concisely and highlight various molecular targets to exert a protective effect on DILI.

\section{Introduction}

Drug-induced liver injury (DILI) is a highly recognized issue that leads to withdrawal of the drugs from the market due to its prognosis with significant adverse effects such as asymptomatic transaminitis, acute and chronic hepatitis, cholestasis, and even death. It is caused not only by prescribed medicines such as nonsteroidal anti-inflammatory drugs (NSAIDs), anti-infective drugs, and anticancer drugs but also by herbal and dietary supplements (HDS) $[1,2]$, particularly acetaminophen (APAP), which is a crucial cause of acute liver failure (ALF) [3].

Acute DILI takes about three months to develop. The type of injured target cells set helps to classify acute DILI into three types using $R=$ (ALT patient/ULN)/(ALP patient/ ULN). These types include (1) hepatocellular type: ALT $\geq 3$ ULN and $R \geq 5$, (2) cholestatic type: ALP $\geq 2$ ULN and $R \leq 2$, and (3) hepatocellular-cholestatic mixed type: ALT $\geq 3$ ULN, $\mathrm{ALP} \geq 2 \mathrm{ULN}$, and $2<R<5$ [1]. DILI is the frequent cause of ALF and accounts for $20-40 \%$ of all instances of fulminant 
hepatic failure throughout the United States and Europe $[4,5]$. The incidence of DILI is as low as 1 in 10,000 to 100,000 [6]. It is difficult to find any particular drug ascribed to hepatic injury. Recent studies indicated the involvement and association of multiple factors in developing DILI. These DILI inducing factors include various environmental factors [1], genetic background [7], drug types [8], age [9], gender $[10]$, pregnancy [11], and underlying liver diseases $[12,13]$.

The in-time diagnosis of DILI is a big challenge. The viral diseases (hepatitis A, B, C, and E virus, cytomegalovirus, and Epstein-Barr virus), autoimmune (antinuclear antibody and antismooth muscle antibody), and metabolic (Wilson's disease and $\alpha 1$-antitrypsin deficiency) disorders mostly mimic any form of acute hepatocellular DILI [14]. Therefore, it is necessary to exclude other liver diseases before clinical diagnosis and treatment of DILI. The frequently used tool for DILI diagnosis is the Roussel Uclaf causality assessment method (RUCAM) [15], which remains highly rational, comprehensive, and convenient. To date, the severity of acute DILI can be classified into five grades, namely, grade $0-5$, where 0 is no liver injury, 1 is mild liver injury, 2 is moderate liver injury, 3 is severe liver injury, 4 is acute liver failure, and 5 is lethal [16].

Different people who are exposed to certain potential hepatotoxic drugs may react differently. The tolerators or nonsusceptible will not have the clinical manifestation of DILI. Mild and transient liver injury can recover naturally after withdrawal of the toxic drug. In certain serve liver injury cases, timely clinical salvage is also needed. Isoflavones are polyphenolic compounds having strong bioactivities primarily found in fruits, flowers, and vegetables. Moreover, isoflavones have been reported to possess estrogen-like effects and hence often called phytoestrogens [17]. The isoflavones have drawn much attention and are considered promising molecules to cure cancer, liver injury, neurodegenerative diseases, osteogenic issues, cardiovascular diseases, and hematotoxicity problems [18-20]. Common isoflavones (biochanin A, formononetin, and genistin) are often found as glycosides are also recognized as mild antioxidants, whereas gut flora help convert these glycosides into aglycones [21]. The isoflavones found in soy are reported to replenish the progress of acetaminophen-mediated hepatotoxicity in vivo by ameliorating the glutathione levels [22]. The isoflavones protect against fatty liver disease (FLD) through multiple cellular pathways involving the $\beta$-oxidation of fatty acid, biosynthesis of lipids, and modulation of reactive oxygen species (ROS). Moreover, aldose reductase (AR)/polyol pathway leads to the development of FLD by modulating the production of fructose in liver, peroxisome proliferator-activated receptor PPAR- $\alpha$ protein, cytochrome P450 (CYP)2E1 expression, and inflammatory factors expressed by certain endotoxins released by the gut flora. The isoflavones have been reported as potent AR inhibitors and hence reduce the onset of FLD [23]. The presence of complex compounds such as biochanin A, calycosin, daidzein, genistein, kakkalide, and tectorigenin in isoflavones made it a vital plant compound bearing various pharmacological properties. Herein, we will elaborate on the updated pharmacological activities of those isoflavones being applied in DILI.

\section{Pathophysiology of DILI}

The nature of DILI is highly complex pathogenesis work in a sequence of effects or simultaneous effects through series of mechanisms along with numerous risk factors, which has not been yet fully explained yet. During drug metabolism, drugs arrive in liver cells via blood circulation, and hence initial drug transport into the hepatocytes begun via influx transporters [24]. The drug in the form of the parent drug is metabolized by phase I and II drug-metabolizing enzymes (DMEs) and products of many reactive metabolites [25]. Phase I metabolism mainly includes cytochrome P450-1 and P450-2 (CYP1 and CYP2) and flavin-containing monooxygenases (FMO) [26]. Human cytochrome P450 2E1 (CYP2E1) metabolizes various drugs, including antitubercular compounds, alcohol, and anesthetics [27]. The mutation in phase I DME genes is a common factor causing the quick incidences of adverse drug reactions (ADRs), which altered the activity of proteins during drug metabolism to induce liver injury [28]. The parent drug, reactive metabolites, and heavier products will then discharge into the bile by efflux transporters [24]. Through phase II DMEs, reactive metabolites with a heavy side chain will be deactivated as toxic compounds [25]. The action of phase II DMEs is to replace the reactive aldehyde and alcohol functional groups with more significant but less reactive functional groups. Conjugated metabolites can be produced and lead the drug to get out in excretion and toxicity via the efflux transporters. In the meantime, conjugated metabolites can also create specific protein adducts that participate in reactive oxygen species- (ROS-) mediated oxidation to damage the cells. While both phases I and II DMEs can clear the parent drug and drugs metabolic products, the phase II metabolized drugs are the most stable and common form of the drug metabolism, with a general tendency to cause cholestasis and DILI during the clearance [29].

\section{Potential Damage and Mechanism in DILI}

3.1. DILI and Reactive Metabolites. During phase I reactions, biotransformation of the parent drug involves necessary steps to complete the subsequent phases of detoxification, which altered the compound highly hydrophilic. The parent drug turned to reactive metabolites in phase I metabolism by adding certain functional groups such as hydroxyl, carboxyl, amino, or thiol groups [25]. These functional groups are said to be highly reactive with proteins compared to the parent drug. ROS and reactive nitrogen species (RNS) that create these reactive metabolites are produced by the interaction of drugs with the proteins and lipid at cellular membranes through oxidative stress [30,31]. Moreover, they can disrupt the cellular redox homeostasis that leads to lymphocytesignaled apoptosis [31].

Furthermore, it can also lead to the development of inflammations by releasing proinflammatory cytokines [32]. In addition, the upregulation of AMP-activated protein kinase (AMPK) and the overexpression of Forkhead box $\mathrm{O} 1$ (FoxO1), which was inhibited by drugs, can increase the 
synthesis of triglycerides (TG). Fatty acid biosynthesis aids in the occurrence of hepatic steatosis, hepatic fibrosis, and even hepatocellular carcinoma [33].

3.2. DILI Induces Mitochondrial Hazards. Mitochondria are cellular energy suppliers, if damaged, resulting in apoptosis and/or hepatic necrosis, even leading to the activation of the apoptotic signaling pathway if the mitochondrial damage exceeds a specific limit [34]. Excessive ROS are produced during the process of DILI. The interaction between ROS and the hepatic mitochondrial membranes is a vital factor of oxidative stress. For example, D-galactosamine/lipopolysaccharide (D-GalN/LPS), a high production level of MDA and an end-product of lipid hydroperoxide (LPO), may lead to decreased membrane fluidity and hence induce severe mitochondrial membranes damage [35]. Few studies have shown that the ethanol treatment can enhance the release of cytochrome $\mathrm{C}$ and apoptosis-inducing factor (AIF), restricted to the mitochondria but released into the cytosol [36]. Cytochrome $\mathrm{C}$ can trigger caspase- 3 activation, which results in apoptosis. At the same time, proapoptotic protein AIF is independent of the caspase pathway and directly induces apoptosis of hepatocytes [31].

Moreover, mitochondrial permeability transition pore (MPTP) plays a significant role in maintaining mitochondrial physiology. In acute severe hemorrhagic shock, ROS sharply increased, leading to the opening of mitochondrial permeability transition pore (MPTP), resulting in the imbalance of $\mathrm{H}^{+}$on the inner mitochondrial membrane, destroying membrane proteins, inhibiting ATP synthesis, and causing mitochondrial swelling [37]. All these may exacerbate necrosis or apoptotic cascades, leading to rapid cell death $[24,38]$.

3.3. DILI Regulates Hepatic Transporters. Drug transporter proteins are crucial factors in clearing reactive metabolites in the liver, intestine, kidney, and brain [39]. According to the mechanism of action and location on the hepatocyte cell membrane, hepatic transporter proteins can be classified into the solute carrier (SLC) and the ATP-binding cassette (ABC) transporter family. Generally, SLC proteins are influx transporters, which bring drugs from the plasma into the cell $[25,40]$, while $A B C$ transporters are efflux transporters, transforming metabolized drugs from the cytoplasm to the cell's exterior in the bile [41].

The transporter protein families in the hepatocytes include organic anion transporting polypeptides (OATPs), organic cation transporters (OCTs), multidrug-resistant proteins (MDRs), bile salt export pump (BSEP), breast cancer resistant proteins (BCRP), and multidrug resistanceassociated proteins (MRPs) [41, 42]. It has been found that OATP1B and sodium-dependent taurocholate cotransporter (NTCP) significantly affect drug transport of all the influx transporters $[39,43]$, while $\mathrm{ABC}$ transport protein (ABCC24 ) is essential for liver cells to clear the chemicals and biliary excretion. The drugs induced mutations in $\mathrm{ABC}$ transporters cannot metabolize drugs to remain inside the hepatocytes and cause impaired canalicular bile flow. This situation generally appears as cholestasis and fatty liver disease [44]. Widely, inhibition of BSEP leads to the accumulation of toxic bile salts in hepatocytes, which are correlated with the incidence of cholestatic liver disease [41]. Therefore, drugs, such as troglitazone, ketoconazole, nefazodone, and lapatinib [45], which express inhibitory effects on BSEP, can have hepatotoxic potential [46]. Furthermore, age and race play an important role in the morbidity of ADRs from variations in the expression of transporter genes, leading to decreased expression of these functional transporters [47].

3.4. DILI Modulates Immunological Response. The immune response is also indispensable in DILI. Long-term drug exposure may cause inflammations in a healthy liver, druginduced autoimmune hepatitis (DIAIH), or acute liver toxicity $[48,49]$. In the adaptive immune response, the drug and its metabolites act as haptens that bind to liver protein cytochrome $\mathrm{p} 450$. The drug-protein adduct is then processed by an antigen-presenting cell (APC), while antigen associates with major histocompatibility complex (MHC) class II molecules. After that, CD4 T-cell is activated, resulting in adaptive immune response, which then triggers CD8 cytotoxic T-cell activation, leading to FasL, TNF- $\alpha$, and other proteins that mediate cell death [50].

The nuclear factor kappa-light-chain enhancer of activated $\mathrm{B}$ cells $(\mathrm{NF}-\kappa \mathrm{B})$ is a dimer protein, which plays an essential role in releasing inflammatory cytokines. It is generally composed of two functional subunits (P65 and $\mathrm{P} 50)$ that bind to its natural inhibiting factor, $\mathrm{I} \kappa \mathrm{B}$, preventing NF-particles from entering the nucleus and targeting downstream associated genes. Toll-like receptor 4 (TLR4)/myeloid differentiation factor 88 (MyD88) receptor receives the irritant cytokines, activates the mitogen-activated protein kinases (MPAK) signaling pathway to induce inflammation, and has three subunits (p38, JNK, and ERK) [51]. The activation of the NF- $\kappa \mathrm{B}$ signaling pathway can increase the risk of inflammation and cell damage. Highmobility group protein box 1 (HMGB1) is reported to play an essential role in sepsis, and it can activate innate immune cells that result in antigen-presenting cells $[52,53]$. Many studies reported that SRT1720 reduces the release of inflammatory cytokines (TNF- $\alpha$ and IL-6) and hence blocks the inflammatory reactions. Additionally, immune-based DILI can also connect with gender, age, race, and current immune state [54].

\section{Health Benefits of Isoflavones}

4.1. As Antioxidants. Oxidative stress, caused by imbalanced cellular ROS levels, is a critical phenomenon in chronic diseases, cardiopathy, and hepatotoxicity. Many studies showed that soybean isoflavones (daidzein and genistein) and red clover isoflavones (biochanin A) potentially protect from DILI [55-57]. Isoflavones play an essential role as dietary antioxidants by scavenging free radicals and deactivating detoxifying enzymes $[57,58]$. Recently, it has been showing that germinated and fermented soybean extracts (GFSE) effectively inhibit the expression of NADPH oxidase 
4 (Nox4), which is responsible for the synthesis of ROS [57-59]. Reduced metabolic activation of drugs by monooxygenase (P450 2E1) system depresses the initial formation of ROS and some intermediate toxic products, resulting in reduced lipid peroxidation [60, 61]. These isoflavones treatments were found to increase the mRNA and protein expression level of CYP2E1 and regulate the JNK/CYP7A1 signaling pathway $[62,63]$. In addition to CYP450, the inhibition of phase II detoxifying enzymes, such as UDPglucuronyltransferase (UGTs), can also have a resistance to oxidative stress [64].

Recently, huge reports highlighted the antioxidant defenses of isoflavones, including both the nonenzymatic (mainly GSH) and enzymatic antioxidant defenses (SOD, CAT, GSP, GST, and GR) $[58,60,65,66]$. Nevertheless, this way has not already been confirmed in the antioxidant protection after GalN administration [67]. Formononetin (FMN) is one of the significant isoflavonoid constituents from red clover, and it showed that the pretreatment of FMN significantly enhanced $\mathrm{Nrf} 2$ protein expression, stimulated mRNA expression of antioxidant enzymes, and restored GSH level and cell viability upon APAP exposure [68]. Nrf2 regulates and induces its downstream heme oxygenase 1 (HO-1) enzyme, which is reported to have antioxidant activity. Biochanin A upregulated the expression of Nrf2 and HO- 1 in a dose-dependent manner and helped resist LPS/DGalN-induced acute liver injury [69].

The dose and route of isoflavones have different effects on resisting oxidative stress. It has been suggested that the efficacy of a high dose of biochanin A (BCA) is lower than a medium dose of $\mathrm{BCA}$, and it is metabolized initially into genistein. Nevertheless, excessive BCA, which was metabolized into genistein and daidzein, could be the primary reason for the high dose of $\mathrm{BCA}$, while $\mathrm{BCA}$ is lower than the medium dose of BCA [57]. Many phytochemicals, such as kakkalide, make contact with intestinal microflora and change it into irisolidone in the alimentary tract. The reports showed that irisolidone was intraperitoneally administered to mice or kakkalide was orally administered to mice. Both exhibited potent hepatoprotective activity. Nevertheless, intraperitoneally administration of kakkalide did not exhibit hepatoprotective activity [70-72].

4.2. Antiapoptosis. Recent studies have shown that high ROS concentrations may contribute to apoptotic cell death and DNA damage. Some studies indicated that puerarin (PR) could inhibit apoptosis due to the suppression of caspase-3 activity and alteration of $\mathrm{Bcl}-2 / \mathrm{Bax}$ ratio, which can alleviate raised lead-induced hepatic dysfunction and histopathologic changes rat liver [73]. Further, a downregulation in Bcl-2 and an upregulation in Bax induced by 7,12-dimethylbenz $[\alpha]$-anthracene (DMBA) treatment were also decreased by daidzein [74]. Genistein treatment, on the other hand, also downregulates Bcl-2 levels [75]. Meanwhile, the metabolite tectorigenin from Puerariae Flos (isoflavones tectorigenin and tectoridin) prevents liver injury because of its inhibited ion of $\beta$-glucuronidase and apoptosis rather than its antioxidant activity [76, 77]. In addition, overexpression of
GSK-3 $\beta$ activates caspase series to activate apoptosis, but puerarin (PR) may inhibit the GSK-3 $\beta$-induced apoptosis by attenuating inflammatory responses via regulating the GSK$3 \beta / \mathrm{NF}-\kappa \mathrm{B}$ pathway [78].

4.3. Anti-Inflammatory and Antifibrosis Effects. With alcohol becoming popular in social life, inflammation is relatively easy to find in most clinical cases, especially alcohol-induced liver damage. The inflammatory responses are mainly linked with the activation of TNF- $\alpha$ mediators and the production of IL- $1 \alpha$, IL- 6 , and released hepatic stellate cells (HSC) following activation to recruit cytokine-mediated immunological attack/lesions.

It is further revealed that isoflavones play an essential role in removing inflammation-related issues. A study found that puerarin $(\mathrm{PR})$ treatment significantly downregulated TLR4 and p38 phosphorylation levels in mouse livers to inhibit Ni-induced oxidative stress and inflammatory responses. However, p38 also can regulate inflammatory response by activating cAMP response element-binding protein (CREB). The TLR4/p38/CREB pathway plays a crucial role in activating transcription factor NF-kB, which plays a fundamental role in regulating gene expression of inflammatory mediators such as PGE2, COX-2, IL-6, and IL8, by activating NF-kB and MAP Kinases [79]. NLRP3 inflammasome is responsible for the processing and secretion of the mature IL- $1 \alpha$. The BCA inhibits thioredoxininteracting protein (TXNIP) expression and the interaction between NLRP3 and TXNIP induced by GalN/LPS, which may be due to the reduced expression of TXNIP [69].

Most of the studies showed a strong relationship between the inflammatory processes and fibrosis. Alcoholic liver disease (ALD) can cause a high death rate, which leaded liver fibrosis, cirrhosis1, and hepatocellular carcinoma [80]. Acetaldehyde, the primary metabolic product of alcohol, activates hepatic stellate cells (HSCs) that play a vital role in liver fibrosis. Herbal medicines have been regarded as new and promising drugs to resist liver fibrosis. It is confirmed that isoflavones (genistein) significantly decreased the extraordinarily high level of TGF- $1 \alpha$ and Smad 3 , which is the activator of HSCs [75]. Tyrosine kinase, one of the factors of HSC activation, is a crucial factor in the proliferation and activation of HSCs. Another study showed that genistein can prevent the activation and proliferation of HSC by inhibiting tyrosine kinase from reducing acute and chronic inflammation and liver fibrosis $[61,65]$. Meanwhile, a study shows that ethyl acetate fraction (Beac) from Butea monosperma bark can inhibit thioacetamide-induced liver cancer and fibrosis via suppression of PI3K/Akt/mTOR pathway [81].

4.4. Antihematotoxicity. Hematotoxicity referred to the influence of drugs on the formation and function of blood, including the inhibition of red and blood cells, platelet count, and hematopoietic function of bone marrow cells. It is illuminated that daidzein pretreatment in rats had a potential beneficial effect on the hematopoietic system by elevating RBCs, Hb levels, and PCV and increasing platelet count against cisplatin-induced hematotoxicity markers. 
TABLE 1: Hepatoprotective effects of isoflavone and extracts.

\begin{tabular}{|c|c|c|c|c|}
\hline Isoflavone and extracts & Drugs & Injury & Mechanism & Refs \\
\hline \multirow{3}{*}{ Biochanin A } & Arsenic & Hepato/hematotoxicity & Increasing free radical scavenging properties & [57] \\
\hline & $\mathrm{CCl} 4$ & Acute liver injury & Increasing antioxidant & {$[60]$} \\
\hline & $\begin{array}{l}\text { LSP/D- } \\
\text { GalN }\end{array}$ & Acute liver injury & Activation of the Nrf2 pathway & {$[69]$} \\
\hline \multirow[t]{2}{*}{ Formononetin } & APAP & Hepatotoxicity & Enhancing Nrf2 activity & {$[68]$} \\
\hline & TAA & Oxidative stress & Reducing ROS and expression of CYP2E1 & {$[62]$} \\
\hline \multirow{3}{*}{ Calycosin } & APAP & NASH & Activating FXR & {$[85]$} \\
\hline & HFD & NAFLD & Activating FXR & {$[86]$} \\
\hline & $\mathrm{CCl} 4$ & Acute liver injury & $\begin{array}{l}\text { Activating FXR/increasing expressions of STAT3, Bcl-xl, and } \\
\text { SOCS3 }\end{array}$ & {$[87]$} \\
\hline \multirow{4}{*}{ Daidzein } & $\begin{array}{l}\text { LSP/D- } \\
\text { GalN }\end{array}$ & Hepatic failure & $\begin{array}{l}\text { Suppressing production of TNF- } \alpha \text {; increasing caspase-3 } \\
\text { activity }\end{array}$ & {$[56]$} \\
\hline & D-GalN & Liver injury & Activating PG-liposome-based system & {$[67]$} \\
\hline & DMBA & Liver oxidative injury & $\begin{array}{l}\text { Activation of antioxidant enzymes and Bcl-2; upregulation of } \\
\text { caspase- } 3 \text { and Bax }\end{array}$ & {$[74]$} \\
\hline & Cisplatin & Hepato/hematotoxicity & $\begin{array}{l}\text { Increasing free radical scavenging properties; reducing bone } \\
\text { marrow suppression }\end{array}$ & {$[82]$} \\
\hline \multirow{5}{*}{ Puerarin } & $\mathrm{CCl} 4$ & $\begin{array}{l}\text { Liver oxidative injury/ } \\
\text { hyperlipidaemia }\end{array}$ & Inhibiting JNK/c-Jun/CYP7A1 pathway/ROS generation & {$[63]$} \\
\hline & Alcohol & Acute liver injury & $\begin{array}{l}\text { Decreasing activity of oxidant enzyme (MDA)/increasing } \\
\text { activity of antioxidant enzymes (SOD and GPX) }\end{array}$ & [66] \\
\hline & Lead & $\begin{array}{l}\text { Liver oxidative injury/ } \\
\text { hyperlipidaemia }\end{array}$ & $\begin{array}{l}\text { Reducing ROS production/hepatic metabolism genes } \\
\text { expression; increasing activation of antioxidant enzymes }\end{array}$ & {$[73]$} \\
\hline & Alcohol & Chronic liver injury & Inhibiting GSK- $3 \beta / \mathrm{NF}-\kappa \mathrm{B}$ pathway & {$[78]$} \\
\hline & Nickel & $\begin{array}{l}\text { Liver oxidative stress/ } \\
\text { immunotoxicity }\end{array}$ & Inhibiting TLR4/p38/CREB pathway & [79] \\
\hline \multirow{5}{*}{ Genistein } & TAA & $\begin{array}{l}\text { Liver inflammatory/ } \\
\text { fibrosis }\end{array}$ & Inhibiting tyrosine kinase/activation of HSC & {$[61]$} \\
\hline & APAP & Liver injury & Increasing expression of UGTs; inhibiting CYP2E1 & {$[64]$} \\
\hline & Alcohol & Hepatic injury/fibrosis & $\begin{array}{c}\text { Increasing activities of ADH and ALDH; downregulating } \\
\text { expression of TIMP-1, MMP-2, and Bcl-2 }\end{array}$ & {$[75]$} \\
\hline & Endotoxin & Shock/MODS & $\begin{array}{l}\text { Decreasing expression of iNOS and COX-2 protein; } \\
\text { attenuating the vascular hyporeactivity to NA; inhibiting the } \\
\text { activity of protein tyrosine kinase }\end{array}$ & {$[84]$} \\
\hline & Fructose & NAFLD & $\begin{array}{c}\text { Activating AMPK and suppressing SREBP-1 cleavage } \\
\text { processing and de novo lipogenesis in hepatocytes } \\
\text { Orally administered kakkalide and intraperitoneally }\end{array}$ & {$[88]$} \\
\hline Kakkalide & Ethanol & Hepatic injury & $\begin{array}{l}\text { administered irisolidone have the protective effect; kakkalide } \\
\text { can be metabolized to form the bioactive irisolidone by } \\
\text { intestinal microflora }\end{array}$ & {$[71,72]$} \\
\hline \multirow[b]{2}{*}{ Tectorigenin } & TBHQ & Apoptosis in liver injury & Inhibiting $\beta$-glucuronidase activity & {$[77]$} \\
\hline & Ethanol & Liver steatosis & $\begin{array}{l}\text { Increasing expression of PPAR- } \alpha \text {; ameliorating mitochondrial } \\
\text { function }\end{array}$ & [89] \\
\hline Flemingia macrophylla & $\mathrm{CCl} 4$ & Acute hepatotoxicity & $\begin{array}{c}\text { Inhibiting ROS generation; preventing lipid peroxidation; } \\
\text { strengthening antioxidant systems }\end{array}$ & {$[58]$} \\
\hline $\begin{array}{l}\text { Fraxin isolated from } \\
\text { Acer tegmentosum }\end{array}$ & $\mathrm{CCl} 4$ & Acute hepatotoxicity & Lowering AST and ALT & [91] \\
\hline GFSE & TBHQ & Hepatotoxicity & $\begin{array}{c}\text { Downregulating NOX4; upregulating the mRNA levels of } \\
\text { antioxidant enzymes }\end{array}$ & {$[59]$} \\
\hline Butea monosperma & TAA & Liver injury & Deregulation of PI3K/Akt/mTOR signaling & {$[81]$} \\
\hline
\end{tabular}

AST: aspartate aminotransferase; ALT: alanine aminotransferase; ADH: alcohol dehydrogenase; ALDH: aldehyde dehydrogenase; APAP: acetaminophen; CCl4: carbon-tetrachloride; CYP2E1: cytochromeP450, family 2, subfamily E, polypeptide1; COX: cyclooxygenase; DMBA: 7,12-dimethylbenz[a]-anthracene; FXR: farnesoid X receptor; GFSE: germinated and fermented soybean extract; GSK-3 $\beta / N F-\kappa$ B pathway: glycogen synthase kinase- $3 \beta /$ nuclear factor kappa-B pathway; HFD: high-fat diet; iNOS: inducible nitric oxide synthase; JNK/c-Jun/CYP7A1 pathway: c-Jun NH2-terminal kinase/c-Jun protein/ cholesterol 7a-hydroxylase pathway; LSP/D-GalN: lipopolysaccharide/D-galactosamine; LPO: lipid peroxides; MMP-2: matrix metalloproteinases; MODS: multiple organ dysfunction syndrome; NASH: nonalcoholic steatohepatitis; NAFLD: nonalcoholic fatty liver disease; NOX4: nicotinamide adenine dinucleotide phosphate oxidase 4; PI3K/Akt/mTOR: phosphatidylinositol 3-kinase/Akt/mammalian; SOCS3: suppressor of cytokine signaling 3; STAT3: signal transducer and activator of transcription 3; TAA: thioacetamide; TLR4/p38/CREB pathway: Toll-like receptor 4/cAMP response element-binding protein; TIMP-1: tissue inhibitors of metalloproteinases; TBHQ: tert-butyl hyperoxide. 
Therein, enhancement of erythrocyte count by daidzein may be linked to either stimulation of erythropoiesis or prevention of bone marrow cell inhibition or decreasing cholesterol effects $[82,83]$. In addition, the antihematotoxicity protective effect of isoflavones can also be related to the dose administered. Fine shreds of evidence claimed that only lowdose BCA had a significant ameliorative effect on arsenicinduced hematopoietic, especially the RBC indices [57].

4.5. Anticirculatory Failure and Organ Dysfunction Effects. The progression of anticirculatory failure (shock) to multiple organ dysfunction syndromes (MODS) is related to increased mortality. With the increasing number of organ failures, mortality is also progressively increased. The endotoxic shock in rats was regarded as the anticirculatory failure model. Different kinds of isoflavones have different effects on these models. It was found that genistein attenuated the vascular hyperactivity to noradrenaline (NA), but it did not reduce the hypotension elicited by LPS. Daidzein did not revive the circulatory failures caused by LPS. Additionally, both tyrphostin and genistein improve the liver and pancreatic injuries, hypoglycemia, and lactic acidosis caused by LPS, while daidzein did not prevent organ dysfunction or LPS-mediated lactic acidosis. Genistein reduced the high level of LPS-induced TNF- $\alpha$ and suppressed the expression of iNOS and COX-2 proteins and activity in the lung, but not daidzein [84]. Thus, genistein can be regarded as a novel and effective drug to improve LPS-induced circulatory failures.

4.6. Antisteatohepatitis. The risk of steatohepatitis has a rapid increase over the last decade because of the bountiful lifestyles and poor eating habits. Nonalcoholic steatohepatitis (NASH) and alcoholic steatohepatitis are two widespread types of steatohepatitis in clinic cases. NASH, being the frequent form of chronic liver disease, may be induced from nutritional (high-fat diet and high fructose diet), genetic, and immunologic factors, which cause disorders in lipid metabolism and hepatic fibrosis. It is further reported that calycosin is a main active component isolated from Radix Astragali and has strong anti-NASH activity. Calycosin treatment has significantly changed the liver histopathology, hepatocytes apoptosis, liver bile acid overload, and hepatocyte mitosis by inhibiting triglycerides (TG) synthesis, increasing fatty acid $\beta$-oxidation, and inhibiting HSCs activation, which helps prevent triglyceride accumulation and fibrosis [85]. It has been shown that calycosin that prevents HFD-induced NAFLD may be attributed to farnesoid X receptor (FXR- $\alpha$ and NR1H4) activation that modulates lipid metabolism and restoration of glucose homeostasis [86].

Furthermore, few studies reported that the protective effects of calycosin are in association with FXR activation and STAT3 phosphorylation. The phosphorylated STAT3 can activate the expressions of Bcl-xl and suppressor of cytokine signaling 3 (SOCS3), which can reduce NASH by preventing liver apoptosis and hemorrhagic necrosis [87]. In addition, it was also found that the treatment of sophoricoside, an isoflavone glycoside (genistein- $4^{\prime}-\mathrm{O}$ $\beta$-D-glucopyranoside), decreased the hepatic cholesterol and triglyceride levels and serum low-density lipoproteincholesterol (LDL) and apolipoprotein-B levels and elevated the serum high-density lipoprotein-cholesterol (HDL) and apolipoprotein-A1 levels [88]. Alcoholic steatosis may progress to hepatitis and fibrosis, which eventually leads to liver cirrhosis. Tectoridin, an isoflavone glycoside from the flower of Pueraria lobata, protected against ethanol-induced liver steatosis mainly through decreasing the expression of $\operatorname{PPAR} \alpha$ and its downstream target genes and ameliorating mitochondrial functions [89].

4.7. Antihyperlipidemia. Puerarin is a potent isoflavone, which seems to be a potential hepatoprotective drug used to inhibit hyperlipidemia, which can decrease serum cholesterol, triglycerides (TG), and LDL and increase HDL level. Hepatic HMGR and hepatic CYP7A1 are peroxisomal enzymes sequentially used in rate-limiting steps during cholesterol biosynthesis and the biosynthesis of bile acids formation. It has been shown that puerarin remarkably inhibited hyperlipidemia by influencing the expression of hepatic lipid biosynthesis and metabolic-related genes, including cholesterol $7 \alpha$-hydroxylase (CYP7A1), HMGR, and low-density lipoprotein receptors (LDL-R) in the liver of lead treated rats [90]. Moreover, puerarin could also inhibit hyperlipidemia by regulating the JNK/CYP7A1 signaling pathways [63].

\section{Conclusions}

With the improvement of living standards and lifestyles, diseases, especially chronic conditions, have increased rapidly. Among various reasons, mainly lousy eating habits and DILI have become the common pathogenic factors. Therefore, specific therapeutic methods have been eagerly sought by many researchers. Recently, the traditional medicinal herbs got the attention. In current essays, puerarin, calycosin, and red clover contain isoflavones that have substantial effects on improving liver injury and circulatory system diseases. All the studies are summarized to elucidate the roles of isoflavones (Table 1) reported in the last decade and we hope it can offer help and convenience in the process of searching for drug treatments.

\section{Abbreviations}

ALF: $\quad$ Acute liver failure

AIF: Apoptosis-inducing factor

APAP: Acetaminophen

AMPK: $\quad$ AMP-activated protein kinase

ADRs: $\quad$ Adverse drug reactions

ABC: $\quad$ ATP-binding cassette

APC: Antigen-presenting cell

BSEP: The bile salt export pump

BCRP: Breast cancer resistant proteins

BCA: Biochanin A

CYPs: $\quad$ Cytochromes P450 


\begin{tabular}{|c|c|}
\hline CYP2E1: & Human cytochrome P450 2E1 \\
\hline CYP1 and & Cytochromes P450 1 and P450 2 \\
\hline CYP2: & \\
\hline CYP7A1: & Cholesterol 7a-hydroxylase \\
\hline CREB: & CAMP response element-binding protein \\
\hline D-GalN/LPS: & D-Galactosamine/lipopolysaccharide \\
\hline DILI: & Drug-induced liver injury \\
\hline DMEs: & Drug-metabolizing enzymes \\
\hline DIAIH: & Drug-induced autoimmune hepatitis \\
\hline FoxO1: & Forkhead box O 1 \\
\hline FXR: & Farnesoid X receptor \\
\hline FMN: & Formononetin \\
\hline FMO: & Flavin-containing monooxygenases \\
\hline GFSE: & Germinated and fermented soybean extracts \\
\hline HDS: & Herbal and dietary supplements \\
\hline HO-1: & Heme oxygenase 1 \\
\hline HSC: & Hepatic stellate cells \\
\hline HDL: & High-density lipoprotein-cholesterol \\
\hline HMGB1: & High-mobility group protein box 1 \\
\hline LPO: & Lipid hydroperoxide \\
\hline LDL: & Low-density lipoprotein-cholesterol \\
\hline LDL-R: & Low-density lipoprotein receptors \\
\hline MDA: & Malondialdehyde \\
\hline MPTP: & Mitochondrial permeability transition pore \\
\hline MDRs: & Multidrug-resistant proteins \\
\hline MyD88: & Myeloid differentiation factor 88 \\
\hline MPAK: & Mitogen-activated protein kinases \\
\hline MRPs: & Multidrug resistance-associated proteins \\
\hline MHC: & Major histocompatibility complex \\
\hline MODS: & Multiple organ dysfunction syndrome \\
\hline NTCP: & $\begin{array}{l}\text { Sodium-dependent taurocholate } \\
\text { cotransporter }\end{array}$ \\
\hline NA: & Noradrenaline \\
\hline NASH: & Nonalcoholic steatohepatitis \\
\hline Nrf2: & Nuclear factor erythroid 2-related factor 2 \\
\hline $\mathrm{NF}-\kappa \mathrm{B}:$ & $\begin{array}{l}\text { Nuclear factor kappa-light-chain-enhancer } \\
\text { of activated B cells }\end{array}$ \\
\hline NSAIDs: & Nonsteroidal anti-inflammatory drugs \\
\hline OATPs: & Organic anion transporting polypeptides \\
\hline OCTs: & Organic cation transporters \\
\hline PR: & Puerarin \\
\hline RUCAM: & Roussel Uclaf causality assessment method \\
\hline ROS: & Reactive oxygen species \\
\hline RNS: & Reactive nitrogen species \\
\hline STAT3: & $\begin{array}{l}\text { Signal transducer and activator of } \\
\text { transcription factor } 3\end{array}$ \\
\hline SLC: & Solute carrier \\
\hline SOCS3: & Suppressor of cytokine signaling 3 \\
\hline TG: & Triglycerides \\
\hline TXNIP: & Thioredoxin-interacting protein \\
\hline TLR4: & Toll-like receptor 4 \\
\hline UGTs: & UDP-glucuronyltransferase. \\
\hline
\end{tabular}

\section{Data Availability}

All the data used to support the findings of this study are included in the paper.

\section{Conflicts of Interest}

The authors declare no conflicts of interest.

\section{Acknowledgments}

This research was funded by the Science and Technology project from Jiangxi Education Department (GJJ180694; 2017JZZDXK004; 20185321).

\section{References}

[1] N. P. Chalasani, P. H. Hayashi, H. L. Bonkovsky, V. J. Navarro, W. M. Lee, and R. J. Fontana, "ACG clinical guideline: the diagnosis and management of idiosyncratic drug-induced liver injury," American Journal of Gastroenterology, vol. 109, no. 7, pp. 950-966, 2014.

[2] E. S. Björnsson, O. M. Bergmann, H. K. Björnsson, R. B. Kvaran, and S. Olafsson, "Incidence, presentation, and outcomes in patients with drug-induced liver injury in the general population of Iceland," Gastroenterology, vol. 144, no. 7, pp. 1419-1425, 2013.

[3] M. W. Russo, J. A. Galanko, R. Shrestha, M. W. Fried, and P. Watkins, "Liver transplantation for acute liver failure from drug induced liver injury in the United States," Liver Transplantation, vol. 10, no. 8, pp. 1018-1023, 2004.

[4] H. Ye, L. J. Nelson, M. G. d. Moral, E. Martínez-Naves, and F. J. Cubero, "Dissecting the molecular pathophysiology of drug-induced liver injury," World Journal of Gastroenterology, vol. 24, no. 13, pp. 1373-1385, 2018.

[5] M. D. Aleo, Y. Luo, R. Swiss, P. D. Bonin, D. M. Potter, and Y. Will, "Human drug-induced liver injury severity is highly associated with dual inhibition of liver mitochondrial function and bile salt export pump," Hepatology, vol. 60, no. 3, pp. 1015-1022, 2014.

[6] D. Larrey, "Epidemiology and individual susceptibility to adverse drug reactions affecting the liver," Seminars in Liver Disease, vol. 22, no. 2, pp. 145-156, 2002.

[7] L. Dara, Z.-X. Liu, and N. Kaplowitz, "Mechanisms of adaptation and progression in idiosyncratic drug induced liver injury, clinical implications," Liver International, vol. 36, no. 2, pp. 158-165, 2016.

[8] E. Pukenyte, F. X. Lescure, D. Rey et al., "Incidence of and risk factors for severe liver toxicity in HIV-infected patients on anti-tuberculosis treatment," International Journal of Tuberculosis \& Lung Disease: The Official Journal of the International Union Against Tuberculosis and Lung Disease, vol. 11, pp. 78-84, 2007.

[9] J. H. Hoofnagle and V. J. Navarro, "Drug-induced liver injury: Icelandic lessons,” Gastroenterology, vol. 144, no. 7, pp. 1335-1336, 2013.

[10] F. J. de Abajo, D. Montero, M. Madurga, and L. A. G. Rodriguez, "Acute and clinically relevant drug-induced liver injury: a population based case-control study," British Journal of Clinical Pharmacology, vol. 58, no. 1, pp. 71-80, 2004.

[11] F. Azizi and A. Amouzegar, "Management of hyperthyroidism during pregnancy and lactation," European Journal of Endocrinology, vol. 164, no. 6, pp. 871-876, 2011.

[12] T. J. Davern, N. Chalasani, R. J. Fontana et al., "Acute hepatitis E infection accounts for some cases of suspected drug- 
induced liver injury," Gastroenterology, vol. 141, no. 5, pp. 1665-1672, 2011.

[13] N. Kamar, C. Garrouste, E. B. Haagsma et al., "Factors associated with chronic hepatitis in patients with hepatitis $\mathrm{E}$ virus infection who have received solid organ transplants," Gastroenterology, vol. 140, no. 5, pp. 1481-1489, 2011.

[14] T. Alempijevic, S. Zec, and T. Milosavljevic, "Drug-induced liver injury: do we know everything?" World Journal of Hepatology, vol. 9, no. 10, pp. 491-502, 2017.

[15] G. Danan and R. Teschke, "RUCAM in drug and herb induced liver injury: the update," International Journal of Molecular Sciences, vol. 17, 2015.

[16] V. Usachov, T. J. Urban, T. J. Urban et al., "Prevalence of genetic variants of keratins 8 and 18 in patients with druginduced liver injury," BMC Medicine, vol. 13, no. 1, p. 196, 2015.

[17] W. Oleszek, "Preface dietary phytochemicals and human health," Phytochemistry Reviews, vol. 1, no. 2, pp. 163-166, 2002.

[18] A. Aras, M. Guven, T. Akman et al., "Neuroprotective effects of daidzein on focal cerebral ischemia injury in rats," Neural Regeneration Research, vol. 10, no. 1, pp. 146-152, 2015.

[19] Y. B. Ye, A. L. Chen, W. Lu et al., "Daidzein and genistein fail to improve glycemic control and insulin sensitivity in Chinese women with impaired glucose regulation: a double-blind, randomized, placebo-controlled trial," Molecular Nutrition \& Food Research, vol. 59, no. 2, pp. 240-249, 2015.

[20] J.-M. Guo, B.-X. Xiao, D.-J. Dai, Q. Liu, and H.-H. Ma, "Effects of daidzein on estrogen-receptor-positive and negative pancreatic cancer cellsin vitro," World Journal of Gastroenterology, vol. 10, no. 6, pp. 860-863, 2004.

[21] G. Bultosa, "Functional foods: overview," Reference Module in Food Science, Elsevier, Berlin, Germany, 2016.

[22] Y.-T. Liu, Y.-H. Chen, N. Uramaru et al., "Soy isoflavones reduce acetaminophen-induced liver injury by inhibiting cytochrome P-450-mediated bioactivation and glutathione depletion and increasing urinary drug excretion in rats," Journal of Functional Foods, vol. 26, pp. 135-143, 2016.

[23] L.-X. Qiu and T. Chen, "Novel insights into the mechanisms whereby isoflavones protect against fatty liver disease," World Journal of Gastroenterology, vol. 21, no. 4, pp. 1099-1107, 2015.

[24] A. Schlessinger, M. A. Welch, H. van Vlijmen, K. Korzekwa, P. W. Swaan, and P. Matsson, "Molecular modeling of drugtransporter interactions-an international transporter consortium perspective," Clinical Pharmacology \& Therapeutics, vol. 104, no. 5, pp. 818-835, 2018.

[25] V. Arya and J. J. Kiser, "Role of transporters in drug development," The Journal of Clinical Pharmacology, vol. 56, pp. S7-S10, 2016.

[26] M.-Y. Lee and J. S. Dordick, "High-throughput human metabolism and toxicity analysis," Current Opinion in Biotechnology, vol. 17, no. 6, pp. 619-627, 2006.

[27] C. Matthias, U. Bockmühl, V. Jahnke et al., "Polymorphism in cytochrome P450 CYP2D6, CYP1A1, CYP2E1 and glutathione S-transferase, GSTM1, GSTM3, GSTT1 and susceptibility to tobacco-related cancers," Pharmacogenetics, vol. 8, no. 2, pp. 91-100, 1998.

[28] S. Crettol, N. Petrovic, and M. Murray, "Pharmacogenetics of phase I and phase II drug metabolism," Current Pharmaceutical Design, vol. 16, no. 2, pp. 204-219, 2010.

[29] T. H. Rushmore and A. N. Tony Kong, "Pharmacogenomics, regulation and signaling pathways of phase I and II drug metabolizing enzymes," Current Drug Metabolism, vol. 3, no. 5, pp. 481-490, 2002.

[30] Q. Cheng, Y.-W. Li, C.-F. Yang et al., "Methyl ferulic acid attenuates ethanol-induced hepatic steatosis by regulating AMPK and FoxO1 pathways in rats and L-02 cells," ChemicoBiological Interactions, vol. 291, pp. 180-189, 2018.

[31] A. F. M. Ismail, A. A. M. Salem, and M. M. T. Eassawy, "Hepatoprotective effect of grape seed oil against carbon tetrachloride induced oxidative stress in liver of $\gamma$-irradiated rat," Journal of Photochemistry and Photobiology B: Biology, vol. 160, pp. 1-10, 2016.

[32] D. Yang, X. Tan, Z. Lv et al., "Regulation of Sirt1/Nrf2/TNF- $\alpha$ signaling pathway by luteolin is critical to attenuate acute mercuric chloride exposure induced hepatotoxicity," Scientific Reports, vol. 6, no. 1, p. 37157, 2016.

[33] J. H. Pan, Y. Lim, J. H. Kim et al., "Root bark of ulmus davidiana var: japonica restrains acute alcohol-induced hepatic steatosis onset in mice by inhibiting ROS accumulation," PLoS One, vol. 12, no. 11, Article ID e0188381, 2017.

[34] M. Chen, A. Suzuki, J. Borlak, R. J. Andrade, and M. I. Lucena, "Drug-induced liver injury: interactions between drug properties and host factors," Journal of Hepatology, vol. 63, no. 2, pp. 503-514, 2015.

[35] J. Zhang, L. Xu, L. Zhang, Z. Ying, W. Su, and T. Wang, "Curcumin attenuates D-galactosamine/lipopolysaccharideinduced liver injury and mitochondrial dysfunction in mice," Journal of Nutrition, vol. 144, no. 8, pp. 1211-1218, 2014.

[36] X. Tian, Y. Hu, M. Li et al., "Carnosic acid attenuates acute ethanol-induced liver injury via a SIRT1/p66Shc-mediated mitochondrial pathway," Canadian Journal of Physiology and Pharmacology, vol. 94, no. 4, pp. 416-425, 2016.

[37] P. Li, X. Wang, M. Zhao, R. Song, and K.-S. Zhao, "Polydatin protects hepatocytes against mitochondrial injury in acute severe hemorrhagic shock via SIRT1-SOD2pathway," Expert Opinion on Therapeutic Targets, vol. 19, no. 7, pp. 997-1010, 2015.

[38] P. Guo, H. Pi, S. Xu et al., "Melatonin improves mitochondrial function by promoting MT1/SIRT1/PGC-1 alpha-dependent mitochondrial biogenesis in cadmium-induced hepatotoxicity in vitro," Toxicological Sciences, vol. 142, no. 1, pp. 182-195, 2014.

[39] B. Stieger, "The role of the sodium-taurocholate cotransporting polypeptide (NTCP) and of the bile salt export pump (BSEP) in physiology and pathophysiology of bile formation," Handbook of Experimental Pharmacology, vol. 201, pp. 205259, 2011

[40] L. Chedik, A. Bruyere, and O. Fardel, "Interactions of organophosphorus pesticides with solute carrier (SLC) drug transporters," Xenobiotica, vol. 49, no. 3, pp. 363-374, 2019.

[41] S. Dawson, S. Stahl, N. Paul, J. Barber, and J. G. Kenna, "In vitro inhibition of the bile salt export pump correlates with risk of cholestatic drug-induced liver injury in humans," Drug Metabolism and Disposition, vol. 40, no. 1, pp. 130-138, 2012.

[42] J. H. Chang, E. Plise, J. Cheong, Q. Ho, and M. Lin, "Evaluating the in vitro inhibition of UGT1A1, OATP1B1, OATP1B3, MRP2, and BSEP in predicting drug-induced hyperbilirubinemia," Molecular Pharmaceutics, vol. 10, no. 8, pp. 3067-3075, 2013.

[43] M.-K. Choi, H. J. Shin, Y.-L. Choi, J.-W. Deng, J.-G. Shin, and I.-S. Song, "Differential effect of genetic variants of Na+taurocholate co-transporting polypeptide (NTCP) and organic anion-transporting polypeptide $1 \mathrm{~B} 1$ (OATP1B1) on the uptake of HMG-CoA reductase inhibitors," Xenobiotica, vol. 41, no. 1, pp. 24-34, 2011. 
[44] E. Kis, E. Ioja, Z. Rajnai et al., "BSEP inhibition-in vitro screens to assess cholestatic potential of drugs," Toxicology in Vitro, vol. 26, no. 8, pp. 1294-1299, 2012.

[45] R. E. Morgan, M. Trauner, C. J. van Staden et al., "Interference with bile salt export pump function is a susceptibility factor for human liver injury in drug development," Toxicological Sciences, vol. 118, no. 2, pp. 485-500, 2010.

[46] E. Ulzurrun, C. Stephens, E. Crespo et al., "Role of chemical structures and the $1331 \mathrm{~T}>\mathrm{C}$ bile salt export pump polymorphism in idiosyncratic drug-induced liver injury," Liver International, vol. 33, no. 9, pp. 1378-1385, 2013.

[47] C. M. Hunt, N. A. Yuen, H. A. Stirnadel-Farrant, and A. Suzuki, "Age-related differences in reporting of drug-associated liver injury: data-mining of WHO safety report database," Regulatory Toxicology and Pharmacology, vol. 70, no. 2, pp. 519-526, 2014.

[48] Z. Wu, M. Han, T. Chen, W. Yan, and Q. Ning, "Acute liver failure: mechanisms of immune-mediated liver injury," Liver International, vol. 30, no. 6, pp. 782-794, 2010.

[49] E. Björnsson, J. Talwalkar, S. Treeprasertsuk et al., "Druginduced autoimmune hepatitis: clinical characteristics and prognosis," Hepatology, vol. 51, no. 6, pp. 2040-2048, 2010.

[50] M. Chakraborty, A. M. Fullerton, K. Semple et al., "Druginduced allergic hepatitis develops in mice when myeloidderived suppressor cells are depleted prior to halothane treatment," Hepatology, vol. 62, no. 2, pp. 546-557, 2015.

[51] W. Zhang, L. Yin, X. Tao et al., "Dioscin alleviates dimethylnitrosamine-induced acute liver injury through regulating apoptosis, oxidative stress and inflammation," Environmental Toxicology and Pharmacology, vol. 45, pp. 193-201, 2016.

[52] J. Xie, J. Wan, R. Jiang, H. Lu, X. Peng, and L. Zhang, "Upregulation of Sirt1 in carbon-tetrachloride-induced acute liver injury," Drug and Chemical Toxicology, vol. 36, no. 3, pp. 277-283, 2013.

[53] W. Xu, Y. Lu, J. Yao et al., "Novel role of resveratrol," Shock, vol. 42, no. 5, pp. 440-447, 2014.

[54] J. Li, X. Zhu, F. Liu et al., "Cytokine and autoantibody patterns in acute liver failure," Journal of Immunotoxicology, vol. 7, no. 3, pp. 157-164, 2010.

[55] B. Y. Chang, D.-S. Lee, J.-K. Lee, Y.-C. Kim, H.-K. Cho, and S. Y. Kim, "Protective activity of kudzu (Pueraria thunbergiana) vine on chemically-induced hepatotoxicity: in vitro and in vivo studies," BMC Complementary and Alternative Medicine, vol. 16, no. 1, p. 39, 2016.

[56] S.-H. Kim, J.-H. Heo, Y. S. Kim, S. S. Kang, J. S. Choi, and S.-M. Lee, "Protective effect of daidzin against d -galactosamine and lipopolysaccharide-induced hepatic failure in mice," Phytotherapy Research, vol. 23, no. 5, pp. 701-706, 2009.

[57] A. M. Jalaludeen, W. T. Ha, R. Lee et al., "Biochanin A ameliorates arsenic-induced hepato- and hematotoxicity in rats," Molecules, vol. 21, p. 69, 2016.

[58] P.-C. Hsieh, Y.-L. Ho, G.-J. Huang et al., "Hepatoprotective effect of the aqueous extract of flemingia macrophylla on carbon tetrachloride-induced acute hepatotoxicity in rats through anti-oxidative activities," The American Journal of Chinese Medicine, vol. 39, no. 2, pp. 349-365, 2011.

[59] E. Y. Kim, K.-B. Hong, H. J. Suh, and H.-S. Choi, "Protective effects of germinated and fermented soybean extract against tert-butyl hydroperoxide-induced hepatotoxicity in HepG2 cells and in rats," Food \& Function, vol. 6, no. 11, pp. 3512-3521, 2015.

[60] R. M. Breikaa, M. M. Algandaby, E. El-Demerdash, and A. B. Abdel-Naim, "Biochanin A protects against acute carbon tetrachloride-induced hepatotoxicity in rats," Bioscience, Biotechnology, and Biochemistry, vol. 77, no. 5, pp. 909-916, 2013.

[61] D. O. Saleh, G. A. R. Abdel Jaleel, S. A. El-Awdan, F. Oraby, and M. Badawi, "Thioacetamide-induced liver injury: protective role of genistein," Canadian Journal of Physiology and Pharmacology, vol. 92, no. 11, pp. 965-973, 2014.

[62] L. Jian, L. Xin, M. Yufang, and H. Yifan, "Protective effect of calycosin-7-O-beta-D-glucopyranoside against oxidative stress of BRL-3A cells induced by thioacetamide," Pharmacognosy Magazine, vol. 11, pp. 524-532, 2015.

[63] J.-Q. Ma, J. Ding, H. Zhao, and C.-M. Liu, "Puerarin attenuates carbon tetrachloride-induced liver oxidative stress and hyperlipidaemia in mouse by JNK/c-Jun/CYP7A1 pathway," Basic and Clinical Pharmacology and Toxicology, vol. 115, no. 5, pp. 389-395, 2014.

[64] Y.-J. Fan, Y. Rong, P.-F. Li et al., “Genistein protection against acetaminophen-induced liver injury via its potential impact on the activation of UDP-glucuronosyltransferase and antioxidant enzymes," Food and Chemical Toxicology, vol. 55, pp. 172-181, 2013

[65] N. Kuzu, K. Metin, A. F. Dagli et al., "Protective role of genistein in acute liver damage induced by carbon tetrachloride," Mediators of Inflammation, vol. 2007, Article ID 36381, 6 pages, 2007.

[66] M. Zhao, Y.-Q. Du, L. Yuan, and N.-N. Wang, "Protective effect of puerarin on acute alcoholic liver injury," The American Journal of Chinese Medicine, vol. 38, no. 2, pp. 241-249, 2010.

[67] M. C. Y. Wong, B. Portmann, R. Sherwood et al., "The cytoprotective effect of $\alpha$-tocopherol and daidzein against d-galactosamine-induced oxidative damage in the rat liver," Metabolism, vol. 56, no. 7, pp. 865-875, 2007.

[68] F. Jin, C. Wan, W. Li et al., "Formononetin protects against acetaminophen-induced hepatotoxicity through enhanced NRF2 activity," PLoS One, vol. 12, no. 2, Article ID e0170900, 2017.

[69] X. Liu, T. Wang, X. Liu et al., "Biochanin A protects lipopolysaccharide/D-galactosamine-induced acute liver injury in mice by activating the Nrf2 pathway and inhibiting NLRP3 inflammasome activation," International Immunopharmacology, vol. 38, pp. 324-331, 2016.

[70] H.-U. Lee, E.-A. Bae, and D.-H. Kim, "Hepatoprotective effects of irisolidone on tert-butyl hyperoxide-induced liver injury," Biological and Pharmaceutical Bulletin, vol. 28, no. 3, pp. 531-533, 2005.

[71] T. Yamazaki, Y. Nakajima, Y. Niho et al., "Pharmacological studies on puerariae flos III: protective effects of kakkalide on ethanol-induced lethality and acute hepatic injury in mice," Journal of Pharmacy and Pharmacology, vol. 49, pp. 831-833, 1997.

[72] Y.-O. Han, M. J. Han, S.-H. Park, and D.-H. Kim, "Protective effects of kakkalide from flos puerariae on ethanol-induced lethality and hepatic injury are dependent on its biotransformation by human intestinal microflora," Journal of Pharmacological Sciences, vol. 93, no. 3, pp. 331-336, 2003.

[73] C.-M. Liu, J.-Q. Ma, and Y.-Z. Sun, "Puerarin protects the rat liver against oxidative stress-mediated DNA damage and apoptosis induced by lead," Experimental \& Toxicologic Pathology, vol. 64, no. 6, pp. 575-582, 2012.

[74] E. J. Choi and G. H. Kim, "Hepatoprotective effects of daidzein against 7, 12-dimetylbenz[a]anthracene-induced oxidative stress in mice," International Journal of Molecular Medicine, vol. 23, pp. 659-664, 2009. 
[75] Q. Huang, R. Huang, S. Zhang et al., "Protective effect of genistein isolated from hydrocotyle sibthorpioides on hepatic injury and fibrosis induced by chronic alcohol in rats," Toxicology Letters, vol. 217, no. 2, pp. 102-110, 2013.

[76] H.-U. Lee, E.-A. Bae, and D.-H. Kim, "Hepatoprotective effect of tectoridin and tectorigenin on tert-butyl hyperoxide-induced liver injury," Journal of Pharmacological Sciences, vol. 97, no. 4, pp. 541-544, 2005.

[77] H.-W. Lee, M.-K. Choo, E.-A. Bae, and D.-H. Kim, " $\beta$-Glucuronidase inhibitor tectorigenin isolated from the flower of Pueraria thunbergiana protects carbon tetrachloride-induced liver injury," Liver International, vol. 23, no. 4, pp. 221-226, 2003.

[78] R. Li, T. Liang, Q. He et al., "Puerarin, isolated from kudzu root (willd.), attenuates hepatocellular cytotoxicity and regulates the GSK- $3 \beta / N F-\kappa B$ pathway for exerting the hepatoprotection against chronic alcohol-induced liver injury in rats," International Immunopharmacology, vol. 17, no. 1, pp. 71-78, 2013.

[79] C.-M. Liu, J.-Q. Ma, S.-S. Liu, Z.-J. Feng, and A.-M. Wang, "Puerarin protects mouse liver against nickel-induced oxidative stress and inflammation associated with the TLR4/p38/ CREB pathway," Chemico-Biological Interactions, vol. 243, pp. 29-34, 2016.

[80] H. Kawaratani, T. Tsujimoto, T. Kitazawa, H. Yoshiji, M. Uemura, and H. Fukui, "Therapeutic effects of cytokine modulator Y-40138 in the rat alcoholic liver disease model," Journal of Gastroenterology and Hepatology, vol. 26, no. 4, pp. 775-783, 2011.

[81] V. Kaur, M. Kumar, P. Kaur, S. Kaur, A. P. Singh, and S. Kaur, "Hepatoprotective activity of butea monosperma bark against thioacetamide-induced liver injury in rats," Biomedicine \& Pharmacotherapy, vol. 89, pp. 332-341, 2017.

[82] S. Karale and J. V. Kamath, "Effect of daidzein on cisplatininduced hematotoxicity and hepatotoxicity in experimental rats," Indian Journal of Pharmacology, vol. 49, pp. 49-54, 2017.

[83] A. Y. Nasr, "Protective effect of aged garlic extract against the oxidative stress induced by cisplatin on blood cells parameters and hepatic antioxidant enzymes in rats," Toxicology Reports, vol. 1, pp. 682-691, 2014.

[84] H. Ruetten and C. Thiemermann, "Effects of tyrphostins and genistein on the circulatory failure and organ dysfunction caused by endotoxin in the rat: a possible role for protein tyrosine kinase," British Journal of Pharmacology, vol. 122, no. 1, pp. 59-70, 1997.

[85] X. Duan, Q. Meng, C. Wang et al., "Calycosin attenuates triglyceride accumulation and hepatic fibrosis in murine model of non-alcoholic steatohepatitis via activating farnesoid X receptor,” Phytomedicine, vol. 25, pp. 83-92, 2017.

[86] X. Duan, Q. Meng, C. Wang et al., "Effects of calycosin against high-fat diet-induced nonalcoholic fatty liver disease in mice," Journal of Gastroenterology and Hepatology, vol. 33, no. 2, pp. 533-542, 2018.

[87] X. Chen, Q. Meng, C. Wang et al., "Protective effects of calycosin against CCl4-induced liver injury with activation of FXR and STAT3 in mice," Pharmaceutical Research, vol. 32, no. 2, pp. 538-548, 2015.

[88] W. Li and Y. Lu, "Hepatoprotective effects of sophoricoside against fructose-induced liver injury via regulating lipid metabolism, oxidation, and inflammation in mice," Journal of Food Science, vol. 83, no. 2, pp. 552-558, 2018.

[89] Y. Xiong, Y. Yang, J. Yang et al., "Tectoridin, an isoflavone glycoside from the flower of Pueraria lobata, prevents acute ethanol-induced liver steatosis in mice," Toxicology, vol. 276, no. 1, pp. 64-72, 2010.

[90] Y. P. Hwang, C. Y. Choi, Y. C. Chung, S. S. Jeon, and H. G. Jeong, "Protective effects of puerarin on carbon tetrachloride-induced hepatotoxicity," Archives of Pharmacal Research, vol. 30, no. 10, pp. 1309-1317, 2007.

[91] B. Chang, Y. Jung, C.-S. Yoon et al., "Fraxin prevents chemically induced hepatotoxicity by reducing oxidative stress," Molecules, vol. 22, no. 4, p. 587, 2017. 